\title{
Diagnostic Sensitivity/Specificity of Preattentive Vision Tests in Glaucoma
}

James Loughman

Technological University Dublin, james.loughman@tudublin.ie

Peter Davison

Technological University Dublin, Peter.Davison@tudublin.ie

Ian Flitcroft

Children's University Hospital, Dublin

Follow this and additional works at: https://arrow.tudublin.ie/otpomart

Part of the Optometry Commons

\section{Recommended Citation}

Loughman, J., Davison, P., Flitcroft, I.: Diagnostic sensitivity/specificity of preattentive vision tests in glaucoma. Optometry and Vision Science, Vol.85, issue no. 7, pp.543-6. 2008. doi:10.1097/

OPX.0b013e31817dd06d

This Article is brought to you for free and open access by ARROW@TU Dublin. It has been accepted for inclusion in Articles by an authorized administrator of ARROW@TU Dublin. For more information, please contact arrow.admin@tudublin.ie, aisling.coyne@tudublin.ie, gerard.connolly@tudublin.ie.

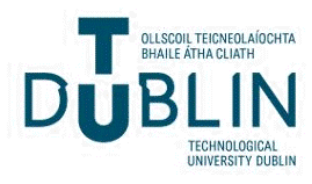




\title{
Diagnostic Sensitivity/Specificity of Preattentive Vision Tests in Glaucoma
}

\author{
JAMES LOUGHMAN, PhD, FAOI, PETER DAVISON, PhD, \\ and IAN FLITCROFT, FRCOphth, DPhil
}

Optometry Department, Dublin Institute of Technology, Republic of Ireland (JL, PD), and Department of Ophthalmology, The Children's University Hospital, Dublin, Republic of Ireland (IF)

\begin{abstract}
Purpose. Damage to the nerve fiber layer or visual pathway might be expected to reduce the efficiency with which the visual system performs analysis of the ever-changing field of vision. The purpose of this article is to provide a further analysis of previously reported data (Loughman J, Davison P, Flitcroft I, Br J Ophthalmol 2007;91:1493-98.) to: (i) determine the sensitivity and specificity of a test of preattentive vision for glaucoma detection and (ii) provide a cutoff performance level that would serve to distinguish glaucoma in early cases.

Methods. Three groups of observers (glaucoma, suspects, and normals) were examined, using computer-generated flicker, orientation, and vertical displacement targets to assess preattentive visual search (PAVS) efficiency. The task required rapid and accurate localization of a singularity embedded in a field of 119 homogenous distractors on either left or right hand side of a computer monitor. All subjects also completed a choice reaction time task.

Results. Receiver operating characteristic curve analysis demonstrates consistently high diagnostic sensitivity and specificity values (significantly above $90 \%$ for all tasks) using the raw PAVS data and also for a novel perceptual search index (which improves the diagnostic capacity of the test). Optimal performance cutoff values for each task were also computed.
\end{abstract}

Conclusions. A test of PAVS efficiency demonstrates high sensitivity and specificity to early glaucoma. Analysis incorporating the perceptual search index confirms the high diagnostic capacity of the test.

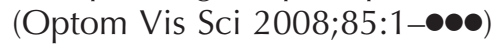

Key Words: preattentive visual search, preattentive vision, glaucoma, flicker, motion displacement, orientation, choice reaction time

$\mathrm{T}$ he ability to process information across the entire visual field simultaneously has long been known ${ }^{1}$ and has been named preattentive vision; it enables the visual system to detect any stimulus (target) which differs sufficiently from all others (distractors) in the visual field; the target exhibits "pop-out" from the distractors and attention is immediately drawn to the target location. An example of preattentive (parallel) and serial search is shown in Fig. 1 below. The three different elements in the left panel pop out due to an orientation difference. The three elements in the right panel differ significantly in appearance but contain the same orientation and line ending information and are therefore not seen preattentively.

Application of the preattentive visual search (PAVS) paradigm to clinical conditions has been relatively recent and confined to investigation of conditions with secondary visual impact, includ- ing Parkinson disease, ${ }^{2}$ senile dementia, and Alzheimer disease. ${ }^{3}$ AQ: 2 Probably the first attempt to investigate the relevance of visual search to primary visual clinical conditions was that of Flitcroft et al., ${ }^{4}$ who devised a set of clinical tests which they found in a preliminary study to correlate with presence of glaucoma. In a recent study, we confirmed the findings of Flitcroft and co-workers in relation to glaucoma..$^{5}$ The purpose of the current article is to present a further statistical analysis of that data. Receiver operating characteristic (ROC) curves were used to (1) generate sensitivity and specificity results and (2) determine the optimal differentiating cutoff normal values for both perceptual search index (PSI) and PAVS results for each target type.

The current preattentive vision test exploits the parallel processing capabilities of the visual system. A test of preattentive vision is inherently different from conventional psychophysical techniques. The 
2 Sensitivity of Preattentive Vision Tests-Loughman et al.

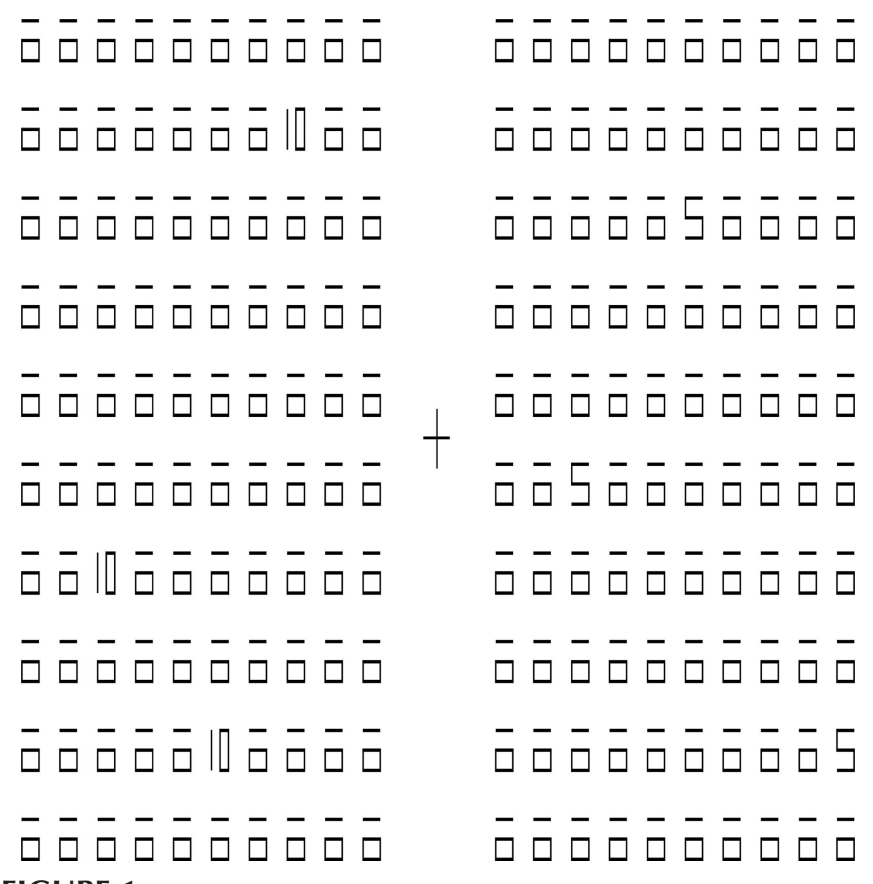

FIGURE 1.

An orientation difference facilitates the pop-out of three targets in the left panel which are readily detected without focused attention. Target differences in the right panel do not pop-out and require serial (foveal) attention to be detected.

parallel processing efficiency of the preattentive system is characteristically assessed by quantification of a subject's capacity to detect feature singularities from a distracting background. As such the subject is presented with multiple targets (in this case 120) and tasked with detection of the single target, which differs from the others in terms of some basic feature such as flicker, motion, or orientation among others. It is reasonable to assume that PAVS requires neural mechanisms across the entire retina to be intact and conditions such as glaucoma may therefore impact on preattentive search efficiency.

\section{METHODS}

The basic test comprises three separate supra-threshold conditionsflicker detection, displacement (motion) detection, and orientation difference detection (an image of the orientation task and full methodological details have been published elsewhere ${ }^{5}$ ). The subject's task was to locate the singular target from among the 119 distractors on either side of the monitor using handheld buttons in a two-alternate forced choice paradigm.

All targets were white with mean luminance of $132 \mathrm{~cd} / \mathrm{m}^{2}$; mean background luminance was $2 \mathrm{~cd} / \mathrm{m}^{2}$ giving a Michelson contrast ratio of 0.97 . The white targets and distractors subtended $0.92^{\circ}$ with a $1.83^{\circ}$ gap between stimuli. The flicker target was a white-filled square box of the above dimensions, square-wave modulated at $16 \mathrm{~Hz}$, and surrounded by identical non-flickering boxes as the distractors.

The displacement target was an empty white box (white lines of width $1 \mathrm{~mm}$, subtending $7 \mathrm{~min}$ of arc, forming a square with an unfilled black center), surrounded by identical stationary boxes as the distractors. The displacement target was displaced vertically by square-wave oscillation at $16 \mathrm{~Hz}$ through an angle of $14 \mathrm{~min}$. The orientation target was the letter $\mathrm{N}$ surrounded by the letter $\mathrm{Z}$ as its distractor; both target and distractor limb widths also subtended 7 min arc. Monitor resolution exceeded that required to present the lines forming the open boxes and $\mathrm{N}$ and $\mathrm{Z}$ targets.

The final test to be completed was a choice reaction time (CRT) test ${ }^{5}$ where the task was to locate a single target from only two visible targets on screen and which was used to determine an index of performance, which we have termed PSI (PSI = PAVS/CRT). The test apparatus (computer, monitor, and test software) and testing routine have previously been described in detail elsewhere. ${ }^{5,6}$

Subjects were informed of the nature of the task and asked for their informed consent. The research protocol followed the tenets of the Declaration of Helsinki and ethical approval was obtained from DIT Research Ethics Committee.

A total of 123 subjects were examined, divided into three agematched groups comprising 41 "normals" (normal IOPs, visual fields, and optic nerve appearance), 41 "glaucoma suspects" (optic nerve appearance characteristic of early glaucoma but without established functional field loss) and 41 "early glaucoma" subjects, including 22 primary open angle glaucoma, 11 normal tension glaucoma, and 8 pseudo-exfoliation glaucoma cases. All subjects were attendees at the ophthalmology department at the Mater Misericordiae hospital in Dublin, and were classified on the basis of optic nerve head appearance, visual fields, and intraocular pressure in a glaucoma clinic, by glaucoma specialists under the guidance of Professor Colm O' Brien. The PAVS examiner was unaware of the diagnosis at the time of testing. All subjects achieved $>90 \%$ accuracy in the detection task eliminating fast-guessing as a complicating factor.

\section{RESULTS}

Standard ROC curve analysis was used to (1) generate sensitivity and specificity results and (2) determine the optimal differentiating cutoff normal values for both PSI and PAVS results for each target type. The statistics software (Stats Direct) plotted sensitivity vs. 1-specificity for a series of cutoff values of both PAVS and PSI; data were inputted for normal and glaucomatous patients only. Optimal normal cutoff values were determined on the basis of maximum sensitivity $\times$ specificity with equal importance assigned to both sensitivity and specificity. Figs. 2 to 4 confirms the F2-4 high diagnostic capacity of the test for all target types. Each figure gives both PAVS and PSI data for one target type.

Table 1 provides a list of sensitivity and specificity values ob- T1 tained for each task and illustrates that for all target types, PSI retains marginally increased sensitivity and specificity over PAVS across each target group indicating that it may provide a slightly better performance index, thus confirming the merit of producing such an index. The "sensitivity $\times$ specificity" index in Table 1 shows the orientation PSI task to yield the most accurate discrimination between groups.

Table 2 gives details of the optimum cutoff values as determined by T2,AQ:4 the analysis. These points are represented by the large open circle and square on the ROC curves for PAVS and PSI data, respectively.

\section{DISCUSSION}

In a clinical setting, and especially in the older population most typically affected by glaucoma, motor, and/or neural factors could potentially influence the accuracy of any interpretation of reac- 


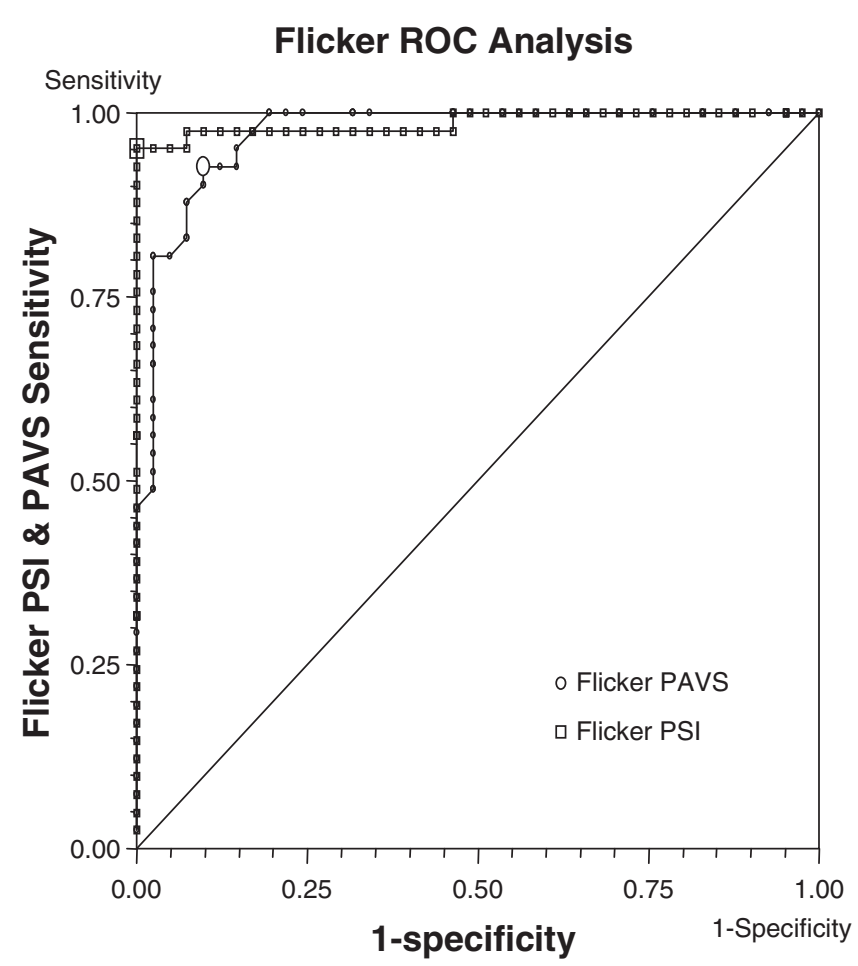

FIGURE 2.

ROC curve for flicker PAVS and PSI, results of normal controls vs. glaucoma subjects.

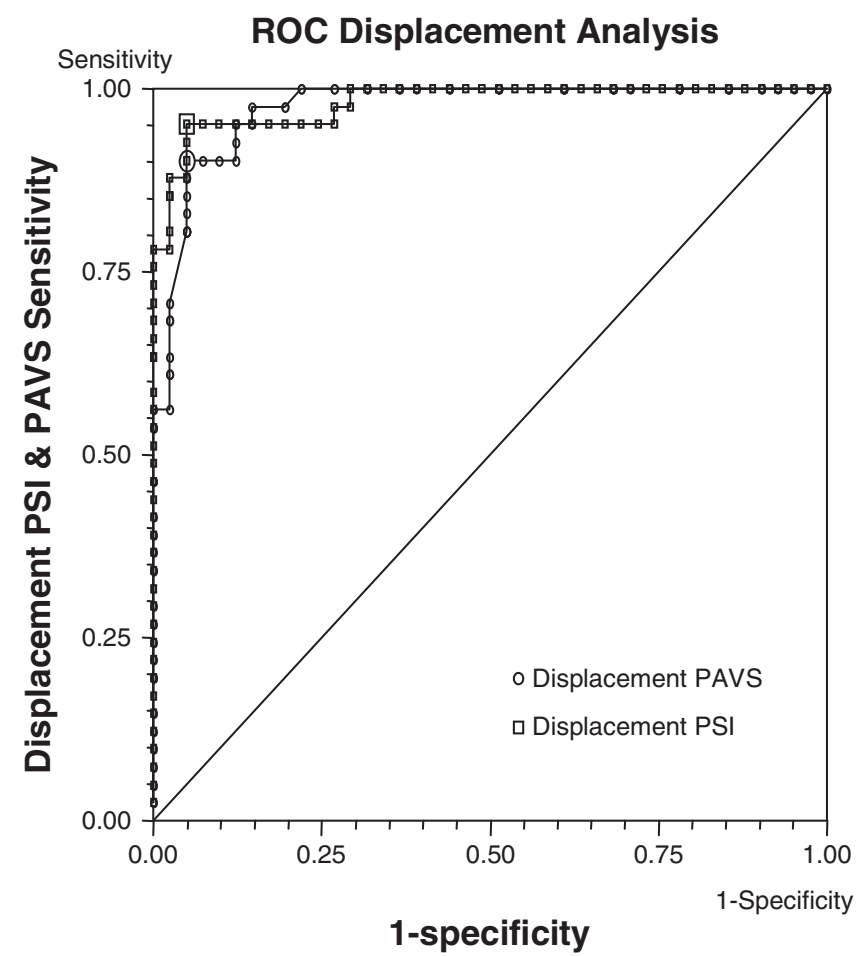

FIGURE 3.

ROC curve for displacement PAVS and PSI, results of normal controls vs. glaucoma subjects.

tion time data. Incorporation of the CRT into the PAVS result giving a PSI index (PAVS/CRT), which renders the test relatively independent of the potential variation with age in the sensory, cognitive, and motor factors that contribute to the individual PAVS reaction time.

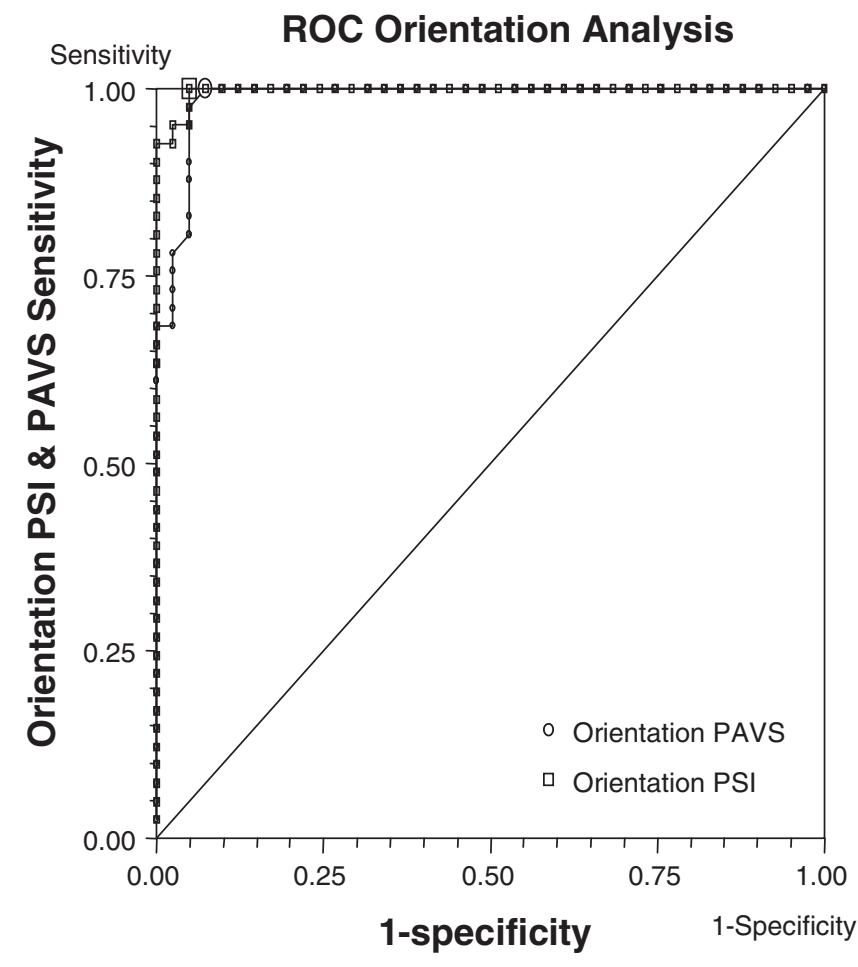

FIGURE 4.

ROC curve for orientation PAVS and PSI, results of normal controls vs. glaucoma subjects.

We have previously reported that this simple step highlights differences in performance efficiency not readily identifiable by analysis of the raw PAVS data. ${ }^{5}$ Furthermore, in the present analysis, Table 1 shows that both sensitivity and specificity are greater for all three tasks using PSI rather than raw PAVS data. With sensitivity and specificity values consistently well above $90 \%$, the results here compare very favorably with alternative functional and structural technologies. ${ }^{8,9}$ Our use of ROC analysis assumed equal weighting of sensitivity and specificity and therefore of type 1 and type 2 errors; in a glaucoma screening environment it would be a matter of clinical judgment whether to modify the weighting.

Differentiating normals from glaucoma is an important factor in determining the clinical value of the test. Equally important, however, is the identification of those patients classified as glaucoma suspects most likely to develop glaucoma. Although longitudinal analysis is essential to determine the test capacity to successfully identify such patients, comparison of the raw suspect data with the determined optimal cutoffs above might give some indication as to those suspects most likely to progress. In total, 14 suspects exceeded the normal PSI criterion on at least one task. Four suspects met the glaucoma PSI criterion on all three tasks. Longitudinal analysis will, however, be required to determine which subjects eventually progress from suspect to glaucoma and whether PAVS is a good prognostic indicator.

It is our contention that analysis of PAVS efficiency such as that achieved in the current device warrants serious consideration as an addition to conventional perimetric methods. The test fulfils numerous important criteria in terms of essential properties of a clinically viable test for glaucoma, including resistance to blur, ${ }^{6}$ simplicity, high diagnostic sensitivity and specificity, as well as a 
4 Sensitivity of Preattentive Vision Tests_Loughman et al.

TABLE 1.

Sensitivity, specificity, area under ROC scores for each task and sensitivity $\times$ specificity scores indicating relative discrimination capacity

\begin{tabular}{|c|c|c|c|c|c|c|}
\hline & $\begin{array}{l}\text { PAVS } \\
\text { flicker }\end{array}$ & $\begin{array}{c}\text { PAVS } \\
\text { displacement }\end{array}$ & $\begin{array}{c}\text { PAVS } \\
\text { orientation }\end{array}$ & $\begin{array}{c}\text { PSI } \\
\text { flicker }\end{array}$ & $\begin{array}{c}\text { PSI } \\
\text { displacement }\end{array}$ & $\begin{array}{c}\text { PSI } \\
\text { orientation }\end{array}$ \\
\hline Sensitivity (\%) & 92.68 & 90.24 & 91.39 & 95.12 & 95.12 & 99.7 \\
\hline Specificity (\%) & 90.24 & 95.12 & 92.68 & 100 & 95.12 & 99.16 \\
\hline Area under ROC & 0.971 & 0.974 & 0.987 & 0.987 & 0.980 & 0.997 \\
\hline Sensitivity $\times$ specificity & 8363.44 & 8583.63 & 8470.03 & 9512 & 9047.81 & $9886.25^{a}$ \\
\hline
\end{tabular}

aMost diagnostic.

\section{TABLE 2.}

Task-specific normal cutoff values for PAVS and PSI

\begin{tabular}{llcc}
\hline & Flicker & Displacement & Orientation \\
\hline Optimum PSI & 1.281 & 1.195 & 1.897 \\
Optimum PAVS (s) & 0.81 & 0.83 & 1.20 \\
\hline
\end{tabular}

patient and practice friendly rapid testing time (screening in $1 \mathrm{~min}$ per eye, maximum test time 6 min per eye in advanced glaucoma).

The current test may benefit from optimization of stimulus parameters and test design features. Longitudinal analysis of glaucoma suspects, and analysis of the effects of other eye disease which may influence PAVS and complicate the clinical diagnosis, deserve further exploration.

Received September 28, 2007; accepted December 20, 2007.

\section{REFERENCES}

1. Neisser U. Cognitive Psychology. New York: Appleton-CenturyCrofts; 1967.

2. Troscianko T, Calvert J. Impaired parallel visual search mechanisms in Parkinson's disease: implications for the role of dopamine in visual attention. Clin Vision Sci 1993;8:281-7.

3. Cormack F, Gray A, Ballard C, Tovee MJ. A failure of 'pop-out' in visual search tasks in dementia with Lewy Bodies as compared to Alz- heimer's and Parkinson's disease. Int J Geriatr Psychiatry 2004;19: 763-72.

4. Flitcroft DI, Doyle A, Eustace P, Migdal C. A new psychophysical approach in glaucoma detection: preattentive vision testing. Invest Ophthalmol Vis Sci 1996;37:S510.

5. Loughman J, Davison P, Flitcroft I. Open angle glaucoma effects on preattentive visual search efficiency for flicker, motion displacement and orientation pop-out tasks. Br J Ophthalmol 2007;91:1493-8.

6. Davison P, Loughman J. Effects of retinal image degradation on preattentive visual search (PAVS) efficiency for flicker, movement and orientation stimuli. Ophthal Physiol Opt 2006;26:456-63.

7. Wolfe JM. Visual attention. In: De Valois KK, ed. Seeing, 2nd ed. San Diego: Academic Press; 2000:335-86.

8. Zangwill LM, Bowd C, Berry CC, Williams J, Blumenthal EZ, Sanchez-Galeana CA, Vasile C, Weinreb RN. Discriminating between normal and glaucomatous eyes using the Heidelberg retina tomograph, GDx nerve fiber analyzer, and optical coherence tomograph. Arch Ophthalmol 2001;119:985-93.

9. Spry PG, Johnson CA, Mansberger SL, Cioffi GA. Psychophysical investigation of ganglion cell loss in early glaucoma. J Glaucoma 2005; 14:11-19.

James Loughman

Optometry Department School of Physics Dublin Institute of Technology Kevin Street, Dublin 8, Republic of Ireland e-mail:james.loughman@dit.ie 


\section{AUTHOR QUERIES}

\section{AUTHOR PLEASE ANSWER ALL QUERIES}

AQ1- Kindly check if the short title is OK as given.

AQ2 - The possessive form has been deleted in all diseases as per the journal style. Kindly check.

AQ3 - Kindly spell out IOP in the text.

AQ4- Kindly check whether Table 2 is OK as edited.

AQ5- Ref. 7 is not cited anywhere in the text. Kindly cite it in order or we will mark it Deleted in Proof. 